\title{
PESQUISA
}

\section{Variação da pressão arterial em trabalhadores de uma siderúrgica}

* Escola Nacional de Saúde Pública - FIOCRUZ - RJ.
Carlos Henrique Klein *

Evandro da Silva Freire Coutinho*

Luiz Antonio Bastos Camacho*

Com o objetivo de estudar a evolução da pressão arterial ao londo da vida ativa de nove categorias de trabalhadores da Companhia Siderírgica Nacional de Volta Redonda (Rio de Janeiro, Brasil), foi analisada uma amostra de 426 homens, através das fichas do serviço médico. Destas fichas, foram coletadas as pressões registradas na admissão na empresa e no último exame de rotina. Cerca de 90\% dos trabalhadores tinham idades entre 20 e 50 anos em qualquer das medidas e o intervalo médio entre estas foi de 13,5 anos para aqueles com pelo menos 5 anos de atividade.

Não foi possivel rejeitar a homogeneidade das idéias de incrementos anuais de sistólica e diastólica entre os grupos ocupacionais. Em relação às variações observadas, houve incrementos estatisticamente significativos na sistólica apenas para os trabalhadores de escritório e almoxarifado, laboratório e controle de qualidade, com pelos menos 5 anos de atividade. Quanto à diastólica, as categorias que sofreram incremento significativo foram escritório $e$ almoxarifado, maquinaria e ocupações especificas da produção, eletro-mecânicas de manutenção, construção civil, transporte e comunicações e trabalhadores não-qualificados.

Também foi analisada a passagem destes trabalhadores de um status de pressão normal para outro de pressão mais elevada (sistólica 140 ou diastólica $90 \mathrm{mmHg}$ ). Com relação à sistólica, apenas os funcionários não-qualificados mudaram de categoria; quanto à diastólica, repetiram-se os achados para incremento de pressão.

O grupo da construção civil destacou-se pelo maior aumento de diastólica entre as duas medidas, ao passo que os administradores e profissionais técnico-cientificos foram os que apresentaram as menores variaçôes de pressão.

Fatores ambientais como ruido, calor, linha de montagem e outros implicados na elevação de pressão arterial estão presentes nesta indústria siderürica.

Os dados apontam maior risco de elevação de pressão arterial em alguns grupos ocupacionais, o que justifica

Recebido para publicação em abordagens diferenciadas pelo serviço médico da empresa.

$24 / 04 / 86$. 


\section{INTRODUÇÃO}

As observaçб̃es acerca de comportamento da pressão arterial no homem indicam ser ela influenciada por diversos fatores constitucionais e ambientais, de natureza ainda pouco conhecida. Ao ambiente de trabalho tem sido atribuída particular importância por conter diversos fatores implicados na etiopatogenia da hipertensão.

São ainda muito raros os estudos feitos no Brasil especificamente sobre o comportamento da pressão arterial de trabalhadores urbanos ${ }^{6,16,19,24}$. Também são escassos aqueles que, apesar de examinarem amostras de populaçб̃es em geral, se preocupam em esmiuçar as relações entre trabalho, a ocupação das pessoas e pressão arterial ${ }^{11,114,15}$.

Todos estes estudos são seccionais, isto é, as medidas de pressão foram feitas numa única ocasião da vida dos examinados. Portanto, as comparações possíveis são aquelas entre grupos ocupacionais ou sociais, num determinado momento. Alguns ${ }^{1,6,14,15}$ concordam com os achados de outros trabalhos feitos em países desenvolvidos ${ }^{10,15,22,23}$ de que há uma correlação negativa entre o status ocupacional e a pressão arterial, tanto sistólica como diastólica. Em outras palavras, as médias mais elevadas de pressão, assim como as maiores prevalências de hipertensão arterial, correspondem aos grupos mais pobres da população, aos trabalhadores não-especializados dos setores secundário e terciário da economia.

Entretanto, pouco se sabe da evolução dos níveis tensionais durante a vida ativa das pessoas, através de observações diretas em pelo menos duas ocasiões. Isto é perfeitamente compreensível, uma vez que, para atingir este fim, seriam necessários estudos longitudinais, de consecução duvidosa, de administração complexa e de custos proibitivos.

Neste trabalho tivemos por objetivo estudar as variações de pressão arterial, sistólica e diastólica, ao longo do tempo de trabalho, em grupos ocupacionais de funcionários da Siderúrgica Nacional de Volta Redonda. Isto foi possível sem a realização de um estudo longitudinal, planejado a propósito, pois lançamos mão dos dados que vinham sendo coletados há muitos anos pelo serviço médico da empresa.

Volta Redonda é um município do estado do Rio de Janeiro no qual está instalada a maior usina siderúrgica do Brasil. Sua população já foi alvo de um estudo epidemioló. gico seccional sobre hipertensão arterial ${ }^{1,15}$. Este estudo foi desenvolvido com recursos do Hospital da Siderúrgica Nacional de Volta Redonda e da Escola Nacional de Saúde Pública - FIOCRUZ. 


\section{MATERIAL E MÉTODOS}

Os dados foram coletados na seção de arquivo do Serviço de Medicina Industrial da Siderúrgica Nacional de Volta Redonda, no segundo semestre de 1979 . $O$ arquivo pesquisado continha as fichas individuais de todos os trabalhadores vinculados diretamente, segundo relação de trabalho com a companhia, em atividade na época.

Inicialmente projetou-se como viável uma amostra de $5 \%$ de todas as fichas, em função dos recursos disponiveis, o que deveria resultar em cerca de 900 fichas. Para tornar a escolha das unidades amostrais aleatória, porém de fácil execução optou-se por um esquema de seleção sistemática. Foi sorteado um dígito terminal do número de matrícula que orientaria a escolha. $O$ dígito sorteado foi o três, assim que, de cada duas fichas com matrícula cujo último número fosse três, uma era selecionada e outra não. Este procedimento foi adotado desde o iń́cio do arquivo até seu esgotamento completo. No final desta primeira etapa, foram escolhidas 918 fichas de Rotina de Inspeção, cada uma correspondendo a um trabalhador.

Foram transcritos para uma ficha especial da pesquisa alguns dados, tais como função, idade, sexo e pressão arterial (sistólica e diastólica). Os dados originais, portanto, não foram coletados para esta pesquisa, mas sim, durante os exames periódicos, de rotina, feitos pelos médicos do serviço. De acordo com as normas, cada trabalhador passava por este exame à cada dois anos e obrigatoriamente ao mudar de função. Este aspecto denuncia o principal problema dos dados que é a ausência de padronização da tomada de informações, o que pode se refletir de forma mais grave justamente nas medidas de pressão arterial. Mais ainda, chamamos atenção para o fato de que a maioria das medidas de pressão foi registrada, na verdade, em centímetros de mercúrio pelos médicos do serviço. Isto pode redundar em menor sensibilidade de detecção de variações, como é nosso propósito.

Foram registrados os dados referentes ao exame de admissão na empresa, ao último exame de reclassificação de função e ao último exame de rotina registrados nas fichas individuais.

Para a análise descrita neste artigo, foram desprezadas todas as fichas em que ocorreu:

a) omissão de quaisquer dos dados de interesse em qualquer exame, por qualquer motivo; a situação mais comum foi aquela em que só havia dados referentes ao exame de admissão;

b) mudança de categoria de função entre o primeiro e o último exame, com exceção dos que passaram de auxiliares 
ou treinandos para a função definitiva;

c) intervalo de tempo entre admissão e último exame menor do que um ano.

Também foram excluídas as mulheres, muito poucas, e, com todas estas restriçð̃es, restaram 426 fichas com dados válidos para esta análise. 0 número de funções registradas na siderúrgica alcança algumas dezenas. $O$ nosso tamanho de amostra final é notoriamente insuficiente para fazer uma análise muito discriminada, de acordo com as funções das variações de pressão. Por isso, resolvemos reunir em categorias chamadas de grupos de ocupações as funçб̃es registradas de acordo com as semelhanças existentes entre elas, de acordo com os critérios atividade, grau de especialização e local de trabalho. Estas categorias também são utilizadas na siderúrgica para classificar seus funcionários.

Foram utilizados os seguintes testes estatísticos: KruskallWallis, para testar diferenças significativas dos incrementos anuais de pressões entre os grupos de ocupações, testes de sinais e de Wilcoxon, para detectar diferenças entre pressões na admissão e no último exame em cada grupo ocupacional, e testes binomiais ou de McNemar, para comparar os níveis de pressão naqueles dois momentos ${ }^{26}$.

A organização e o tratamento dos dados foram feitos em um microcomputador IBM-XT doado pela OMS para a Escola Nacional de Saúde Pública.

\section{RESULTADOS}

Em média, os 426 trabalhadores tinham 23 anos ao serem admitidos e 33 anos no último exame. As idades extremas registradas foram de 13 e 60 anos.

A tabela 1 mostra a distribuição das médias de idades nas duas ocasióes, assim como os tempos médios de trabalho que correspondem aos intervalos de tempo decorridos entre a admissão e o último exame de cada trabalhador. A varia. ção da pressão arterial é fortemente influenciada pelo tempo decorrido entre as medidas; em outras palavras, as pressões se elevam à medida que as pessoas envelhecem ${ }^{7,25}$. Como se pode observar na tabela 1 , há heterogeneidade na distribuição das médias de tempo de trabalho. Para obter um grau mais razoável de homogeneidade dos tempos decorridos, optamos por analisar, em separado, somente aqueles trabathadores com pelo menos cinco anos de trabalho. Na tabela 2 verifica-se que, nesta fração dos trabalhadores $(\mathrm{N}$ total $=$ 302), a distribuição das médias de tempo de trabalho é mais homogênea, permitindo melhor comparabilidade dos grupos ocupacionais em relação às variações de pressão. Ainda, nesta fração mais estável de trabalhadores, a média global 
de trabalho atingiu os treze anos e meio.

TABELA 1

Idade, na admissão e no último exame, e tempo de trabalho, segundo os grupos de ocupações - SNVR, 1979

\begin{tabular}{|c|c|c|c|c|c|c|c|}
\hline \multirow{3}{*}{ Grupos de Ocupações } & \multicolumn{4}{|c|}{$\begin{array}{l}\text { Idade } \\
\text { (anos) }\end{array}$} & \multicolumn{3}{|c|}{$\begin{array}{l}\text { Tempo de Tra- } \\
\text { balho (anos) }\end{array}$} \\
\hline & \multicolumn{2}{|c|}{ Admissão } & \multicolumn{2}{|c|}{ Ult. Exame } & \multirow[b]{2}{*}{$\overline{\mathbf{x}}$} & \multirow[b]{2}{*}{$\mathbf{s}$} & \multirow[t]{2}{*}{$\mathrm{N}$} \\
\hline & $\bar{x}$ & $\mathbf{s}$ & $\bar{x}$ & $\mathbf{s}$ & & & \\
\hline 1 - Administrativas e técnico-científicas & 27,1 & 5,7 & 37,1 & 9,3 & 10,0 & 8,5 & 16 \\
\hline 2 - Escritório e almoxarifado & 21,9 & 4,3 & 33,6 & 9,2 & 11,6 & 8,1 & 49 \\
\hline 3 - Laboratório e controle de qualidade & 22,3 & 4,8 & 32,4 & 7,4 & 10,1 & 6,1 & 23 \\
\hline 4 - Maquinaria e específicas da produção & 23,1 & 4,0 & 35,5 & 7,8 & 12,4 & 7,3 & .56 \\
\hline 5 - Eletro-mecânicas de manutenção & 22,2 & 5,0 & 32,4 & 9,7 & 10,2 & 7,5 & 74 \\
\hline 6 - Construção civil & 23,6 & 3,6 & 38,2 & 8,8 & 14,6 & 8,1 & 22 \\
\hline 7 - Transportes e comunicações & 23,4 & 4,6 & 34,5 & 9,9 & 11,2 & 7,1 & 34 \\
\hline 8 - Prestação de serviços e segurança & 23,4 & 5,9 & 35,7 & 12,3 & 12,3 & 8,8 & 16 \\
\hline $\begin{array}{l}9 \text { - Não-qualificadas (ajudantes, } \\
\text { serventes e trabalhadores) }\end{array}$ & 22,9 & 4,5 & 31,0 & 9,1 & 8,1 & 6,9 & 136 \\
\hline
\end{tabular}

TABELA 2

Idade, na admissão e no último exame, e tempo de trabalho, segundo os grupos de ocupações - SNVR, 1979. Somente para aqueles com 5 e mais anos de trabalho

\begin{tabular}{ccc}
\hline & $\begin{array}{c}\text { Idade } \\
\text { (anos) }\end{array}$ & $\begin{array}{c}\text { Tempo de Tra- } \\
\text { balho (anos) }\end{array}$
\end{tabular}

Grupos de Ocupações

Admissão Ult. Exame

$\mathrm{N}$

$\begin{array}{llllll}\bar{x} & s & \bar{X} & s & \bar{x} & s\end{array}$

1 - Administrativas e técnico-científicas

$25,6 \quad 4,1 \quad 38,6$

$9,9 \quad 13,0$

$8,7 \quad 11$

2 - Escritório e almoxarifado

$\begin{array}{lll}21,8 & 4,5 & 34,7\end{array}$

$9,1 \quad 12,9$

$7,8 \quad 43$

3 - Laboratório e controle de qualidade

$21,4 \quad 4,0 \quad 34,1$

$7,3 \quad 12,7$

$4,9 \quad 17$

4 - Maquinaria e específicas da produção

$23,2 \quad 3,9 \quad 37,3$

$6,8 \quad 14,2$

$6,5 \quad 47$

5 - Eletro-mecânicas de manutenção

$23,0 \quad 5,4 \quad 36,4$

$8,7 \quad 13,4$

$6,7 \quad 52$

6 - Construção civil

$23,8 \quad 3,8 \quad 40,3$

$7,4 \quad 16,5$

$6,9 \quad 19$

7 - Transportes e comunicações

$\begin{array}{lll}24,3 & 4,2 & 37,6\end{array}$

$8,5 \quad 13,2$

$6,5 \quad 27$

8 - Prestação de serviços è segurança

24,8

$6,7 \quad 41,4$

$10,516,6$

$7,1 \quad 11$

9 - Não-qualificadas (ajudantes,

$23,7 \quad 4,9 \quad 36,5 \quad 8,4 \quad 12,8$

$6,1 \quad 75$ serventes.e trabalhadores) 
A tabela 3 apresenta as médias dos incrementos anuais de pressão (pressão no último exame menos a pressão na admissão, dividido pelo tempo de trabalho), sistólica e diastólica, em $\mathrm{mmHg}$, de cada um dos grupos de ocupações. Já que as distribuições originais não eram normais (cada valor era resultado de uma razão), optamos por transformar os valores em postos e aplicar então o teste de Kruskall-Wallis, para decidir se os grupos pertencem a uma mesma popula. ção de trabalhadores. Náo foi possível rejeitar a homogeneidade das médias de incrementos anuais de sistólica.

\section{TABELA 3}

Incrementos anuais das pressões arteriais sistolica e diastólica, segundo os grupos de ocupações SNVR, 1979

\begin{tabular}{|c|c|c|c|c|c|c|c|}
\hline \multirow{3}{*}{ Grupos de Ocupaçð̃es } & \multicolumn{3}{|c|}{ Sistolica } & \multicolumn{4}{|c|}{ Diastolica } \\
\hline & \multicolumn{2}{|c|}{$\begin{array}{l}\text { Val. Originais } \\
\text { (mmHg) }\end{array}$} & \multirow{2}{*}{$\begin{array}{l}\text { Médias } \\
\text { de } \\
\text { Postos }\end{array}$} & \multicolumn{2}{|c|}{$\begin{array}{l}\text { | Val. Originais } \\
\text { (mmHg) }\end{array}$} & \multirow{2}{*}{$\begin{array}{l}\text { Médias } \\
\text { de } \\
\text { Postos }\end{array}$} & \multirow{2}{*}{$\mathbf{N}$} \\
\hline & $\bar{x}$ & s & & $\bar{x}$ & $s$ & & \\
\hline $\begin{array}{l}1 \text { - Administrativas e técnico-científicas } \\
2 \text { - Escritório e almoxarifado } \\
3 \text { - Laboratório e controle de qualidade } \\
4 \text { - Maquinaria e especfficas da produção } \\
\text { 5 - Eletro-mecânicas de manuenção } \\
\text { 6 - Construção civil } \\
7 \text { - Transportes e comunicaçóes } \\
8 \text { - Prestação de serviços e segurança } \\
\text { - Não-qualificadas (ajudantes, } \\
\text { serventes e trabalhadores) }\end{array}$ & $\begin{array}{r}-0,377 \\
0,074 \\
-0,502 \\
0,567 \\
-0,500 \\
0,042 \\
1,111 \\
-0,118 \\
0,107\end{array}$ & $\begin{array}{l}2,168 \\
2,489 \\
6,951 \\
2,683 \\
4,462 \\
2,478 \\
2,609 \\
2,544 \\
5,936\end{array}$ & $\begin{array}{l}175,0 \\
215,0 \\
230,2 \\
216,9 \\
195,3 \\
201,7 \\
240,6 \\
201,1 \\
219,8\end{array}$ & $\begin{array}{r}-0,473 \\
0,197 \\
0,549 \\
0,728 \\
-0,772 \\
0,727 \\
0,826 \\
0,735 \\
0,016\end{array}$ & $\begin{array}{l}2,004 \\
2,216 \\
5,193 \\
2,556 \\
2,718 \\
1,289 \\
2,063 \\
1,884 \\
5,511\end{array}$ & $\begin{array}{l}161,1 \\
222,9 \\
240,8 \\
231,4 \\
177,3 \\
251,3 \\
234,8 \\
225,2 \\
211,2\end{array}$ & $\begin{array}{r}16 \\
49 \\
23 \\
56 \\
74 \\
22 \\
34 \\
16 \\
136\end{array}$ \\
\hline \multicolumn{3}{|l|}{$\begin{array}{l}\text { Estatística Kruskall-Wallis } \\
\text { Significância }\end{array}$} & \multicolumn{2}{|c|}{$\begin{array}{c}6,03 \\
p>0,50\end{array}$} & \multicolumn{3}{|c|}{$\begin{array}{c}15,17 \\
0,10<p>0,05\end{array}$} \\
\hline
\end{tabular}

Já em relação d diastólica é preciso concluir o mesmo, ainda que, neste caso, o valor da estatística esteja bastante próximo ao associado à probabilidade usada como critério de rejeição $(0,05)$.

As tabelas seguintes analisam as variaçбes, propriamente ditas, de pressð̋es nas duas ocasiôes de medida, admissão e último exame, em cada um dos grupos de ocupações.

As tabelas 4 e 5 apresentam os testes de sinais e de Wilcoxon no total e na fração mais estável de trabalhadores, respectivamente, em cada grupo de ocupações. Apesar de que as provas de Wilcoxon incorporem e ainda excedam as propriedades dos testes de sinais, transcrevemos os dados básicos destes últimos a fim de informar os sentidos das diferenças entre pressões (último exame menos admissão). Enquanto o teste dos sinais somente utiliza a informação 
dada pelo sentido da diferença, a prova de Wilcoxon ainda valoriza a magnitude de cada variação.

\section{TABELA 4}

Diferenças de pressōes entre a admissão e o último exame, segundo os grupos de ocupaçōes SNVR, 1979

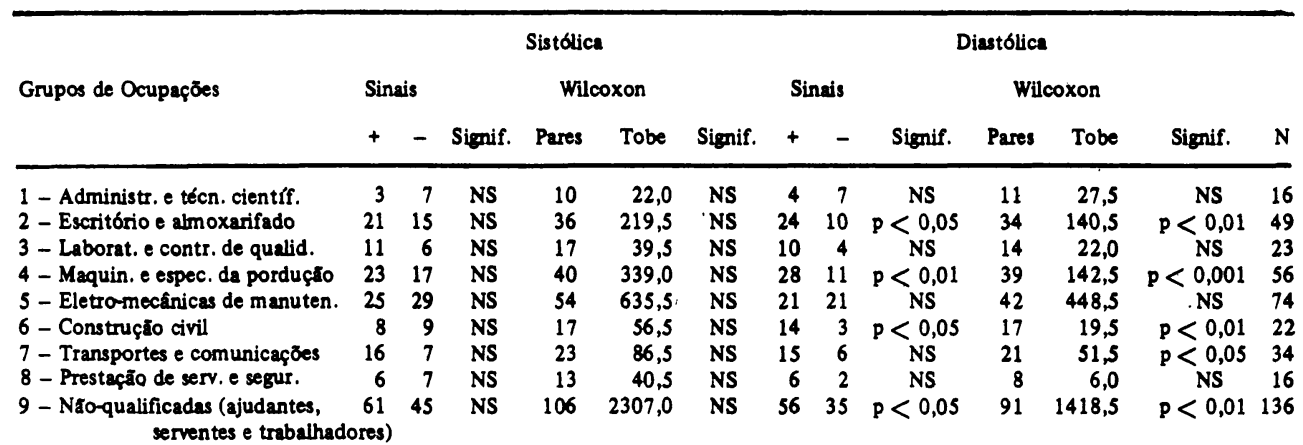

\section{TABELA 5}

Diferenças de pressōes entre a admissāo e o último exame, segundo os grupos de ocupaçōes SNVR, 1979: Somente para aqueles com 5 anos e mais de trabalho

\begin{tabular}{|c|c|c|c|c|c|c|c|c|c|c|c|c|c|}
\hline \multirow{3}{*}{ Grupos de Ocupaçōes } & \multicolumn{6}{|c|}{ Sistólica } & \multicolumn{7}{|c|}{ Dịastólica } \\
\hline & \multicolumn{2}{|c|}{ Sinais } & \multicolumn{4}{|c|}{ Wilcoxon } & \multicolumn{2}{|c|}{ Sinais } & \multicolumn{5}{|c|}{ Wilcoxon } \\
\hline & + & - & Signif. & Pares & Tobe & Signif. & + & - & Signif. & Pares & Tobe & Signif. & $\mathbf{N}$ \\
\hline 1 - Administr. $\mathrm{c}$ técn. científ. & 2 & 4 & NS & 6 & 8,0 & NS & 3 & 4 & NS & 7 & 14,0 & NS & 11 \\
\hline 2 - Escritório e almoxarifado & 20 & 11 & NS & 31 & 140,5 & $\mathrm{p}<0,05$ & 23 & 8 & $\mathrm{p}<0,05$ & 31 & 102,0 & $\mathrm{p}<0,01$ & 43 \\
\hline 3 - Laborat. e contr. de qualid. & 10 & 2 & $\mathrm{p}<0,05$ & 12 & 6,0 & $\mathrm{p}<0,01$ & 7 & 3 & NS & 10 & 9,5 & NS & 17 \\
\hline 4 - Maquin. e espec. da produção & 17 & 15 & NS & 32 & 254,0 & NS & 25 & 8 & $\mathrm{p}<0,01$ & 33 & 85,0 & $\mathrm{P}<0,001$ & 47 \\
\hline 5 - Eletro-mecânicas de manuten. & 18 & 21 & NS & 39 & 358,0 & NS & 20 & 10 & NS & 30 & 130,5 & $\mathrm{p}<0,05$ & 52 \\
\hline 6 - Construção civil & 7 & 7 & NS & 14 & 37,0 & NS & 13 & 3 & $\mathrm{p}<0,05$ & 16 & 18,0 & $\mathrm{p}<0,01$ & 19 \\
\hline 7 - Transportes e comunicações & 13 & 7 & NS & 20 & 71,5 & NŚ & 11 & 5 & NS & 16 & 32,5 & NS & 27 \\
\hline \& - Prestação de serv. e segur. & 4 & 5 & NS & 9 & 19,5 & NS & 4 & 2 & NS & 6 & 4,0 & NS & 11 \\
\hline 9 - Não-qualificadas (ajudantes, & 37 & 22 & NS & 59 & 598,0 & $p<0,05$ & 39 & 14 & $\mathrm{p}<0,001$ & 53 & 320,5 & $\mathrm{p}<0,001$ & 75 \\
\hline
\end{tabular}
serventes e trabalhadores)

Observa-se que não ocorreram alterações sistemáticas da sistólica estatisticamente significativas nos grupos, com exceção da verificada nos funcionários de escritório e almoxarifado, de laboratório e controle de qualidade, e dos nãoqualificados (ajudantes, serventes e outros trabalhadores), mesmo assim entre aqueles com pelos menos cinco anos de atividade. Já na pressão diastólica ocorreram variaçōes significativas nos grupos de escritório e almoxarifado, de maquinaria e específicas da produção, da construção civil, de transportes e comunicaçōes e nos não-qualificados (tabela 4). No caso dos trabalhadores com cinco ou mais anos de atividade, ainda se acrescentou o grupo das ocupações eletro-mecânicas de manutenção, mas não se confirmou o 
de transportes e comunicações (tabela 5). Toda a variação assinalada como estatisticamente significativa se fez no sentido positivo, isto é, a probabilidade da pressão ser mais alta no último exame do que na admissão não pode ser imputada ao acaso.

As tabelas 6 e 7 mostram também as mudanças da pressão, mas de modo diferente. Se nas tabelas anteriores (4 e 5) o critério de comparação era variável em cada indivíduo - a pressão na admissão - nestas últimas o critério é fixo para cada uma das pressões, sistóliça e diastólica. Foram escolhidos como pontos de corte, ou critérios fixos de comparação, os valores de 140 e $90 \mathrm{mmHg}$ para sistolica e diastólica, respectivamente. Estes valores coincidem com aqueles recomendados como os preferidos para classificar as pessoas na categoria limítrofe da normalidade com a hipertensão arterial ${ }^{13}$. Assim, estão fora da normalidade aqueles cujas pressões sejam iguais ou maiores do que as escolhidas como pontos de corte. Alem disso, não optamos pelos tradicionais pontos 160 e $95 \mathrm{mmHg}$, recomendados pela $\mathrm{OMS}^{28}$ para classificar hipertensão definida, já que a grande maioria das pessoas estudadas se encontrava na faixa etária de 20 a 50 anos de idade (90\%), na qual são relativamente raros aqueles que seriam classificados no grupo de hipertensão definida.

\section{TABELA 6}

Comparações entre os ntveis de pressão arterial na admissão com os do último exame, segundo os grupos de ocupaçōes - SNVR, 1979

\begin{tabular}{|c|c|c|c|c|c|c|}
\hline \multirow{2}{*}{ Grupos de Ocupações } & \multicolumn{2}{|c|}{$\begin{array}{c}\text { Sistólica } \\
\begin{array}{c}\text { Admissão/Ult. Exame } \\
(\mathrm{mmHg} / \mathrm{mmHg})\end{array}\end{array}$} & \multirow[b]{2}{*}{ Signific. } & \multicolumn{2}{|c|}{$\begin{array}{r}\text { Diastólica } \\
\text { Admissão/Ult. Exame } \\
(\mathrm{mmHg} / \mathrm{mmHg})\end{array}$} & \multirow[b]{2}{*}{ Signific. } \\
\hline & $\begin{array}{l}<140 /<140 \\
\geqslant 140 /<140\end{array}$ & $\begin{array}{l}<140 / \geqslant 140 \\
\geqslant 140 / \geqslant 140\end{array}$ & & $\begin{array}{l}<90 /<90 \\
\geqslant 90 /<90\end{array}$ & $\begin{array}{l}<90 / \geqslant 90 \\
\geqslant 90 / \geqslant 90\end{array}$ & \\
\hline 1 - Administrativas e técnico-científicas & $\begin{array}{r}13 \\
1\end{array}$ & $\begin{array}{l}1 \\
1\end{array}$ & NS & $\begin{array}{r}12 \\
1\end{array}$ & $\begin{array}{l}2 \\
1\end{array}$ & NS \\
\hline 2 -.Escritório e almoxarifado & $\begin{array}{r}37 \\
3\end{array}$ & $\begin{array}{l}9 \\
-\end{array}$ & NS & $\begin{array}{r}37 \\
2\end{array}$ & 10 & $\mathrm{p}<0,05$ \\
\hline 3 - Laboratório e controle de qualidade & $\begin{array}{r}18 \\
1\end{array}$ & $\overline{2}$ & NS & $\begin{array}{r}2 \\
16 \\
2\end{array}$ & $\overline{4}$ & NS \\
\hline 4 - Maquinaria e especifficas da produção & $\begin{array}{r}39 \\
5\end{array}$ & $\begin{array}{l}6 \\
6\end{array}$ & NS & $\begin{array}{r}35 \\
2\end{array}$ & $\begin{array}{r}17 \\
2\end{array}$ & $\mathrm{p}<0,001$ \\
\hline 5 - Eletro-mecânicas de manutenção & $\begin{array}{r}61 \\
4\end{array}$ & $\begin{array}{l}8 \\
1\end{array}$ & NS & $\begin{array}{r}62 \\
3\end{array}$ & $\begin{array}{l}8 \\
1\end{array}$ & NS \\
\hline 6 - Construção civil & $\begin{array}{r}10 \\
3\end{array}$ & $\begin{array}{l}3 \\
6\end{array}$ & NS & 12 & $\begin{array}{l}8 \\
2\end{array}$ & $\mathrm{p}<0,01$ \\
\hline 7 - Transportes e comunicações & $\begin{array}{r}23 \\
1\end{array}$ & $\begin{array}{l}5 \\
5\end{array}$ & NS & $\begin{array}{r}23 \\
1\end{array}$ & 8 & $\mathrm{p}<0,05$ \\
\hline 8 - Prestação de serviços e segurança & $\begin{array}{r}11 \\
1\end{array}$ & $\begin{array}{l}3 \\
1\end{array}$ & NS & $\begin{array}{r}13 \\
1\end{array}$ & 2 & NS \\
\hline $\begin{array}{l}9 \text { - Não-qualificadas (ajudantes, } \\
\text { serventes e trabalhadores) }\end{array}$ & $\begin{array}{l}82 \\
15\end{array}$ & $\begin{array}{l}26 \\
13\end{array}$ & NS & $\begin{array}{r}100 \\
5\end{array}$ & $\begin{array}{r}23 \\
8\end{array}$ & $\mathrm{p}<0,01$ \\
\hline
\end{tabular}


As tabelas 6 e 7 são compostas por várias de dupla entrada, duas para cada grupo de ocupações, de acordo com o nível de pressão, sistólica ou diastólica, na admissão e no último exame. Para a análise de significância só interessam as células em que ocorreu discordância dos níveis de pressão nas duas ocasiōes. Foram utilizados testes binomiais e McNemar para testar significâncias de mudanças. Novamente todas mudanças significativas se deram no sentido do nível mais elevado de pressão ser observado no último exame, em oposição ao nível inferior na admissão. E mais, os resultados são praticamente idênticos d̀queles obtidos com os testes de Wilcoxon (tabelas 4 e 5). Quer dizer, foram detectadas mudanças significativas nos mesmos grupos apontados anteriormente, nas situações correspondentes (a tabela 6 corresponde à 4 e a 7 d tabela 5). Devem ser registradas somente duas exceções; nos grupos de escritório e almoxarifado, e de laboratório e controle de qualidade, com pelo menos cinco anos de atividade, não foi possível detectar mudanças significativas de sistolica usando-se o método de ponto fixo de comparação (tabela 7):

\section{DISCUSSÃO}

Nos estudos especiais realizados em indústrias ou outros locais de trabalho, quase sempre predomina uma das duas linhas principais de investigação de fatores relacionados com a pressão arterial: a do stress ou a dos agentes físicos e químicos do ambiente.

Pesquisas feitas nos EUA ${ }^{8}$ e na Finlândia ${ }^{3,4}$ são utilizadas para demonstrar que o controle excessivo de emoções, o ocultamento de ressentimentos e impulsos agressivos e a auto-repressão estão estreitamente relacionados com a elevação da pressão arterial, especialmente a diastólica. Entretanto, Mustachi ${ }^{20}$, após ampla revisão a respeito da influência do stress emocional sobre a pressão, acha duvido. sos estes resultados. Este autor conclui que as pessoas reagem de formas muito diferentes, quando não opostas, a um mesmo estímulo, e que, portanto, é dif́cil responsabilizar o desenvolvimento da hipertensão às situações de stress psicológico.

Também já foi observado em trabalhadores manuais, sem especialização, que a pressão arterial era mais elevada naqueles que sofriam mudanças periódicas no seu horário de trabalho ${ }^{21}$. A mudança freqüente de turno de trabalho é comum nos trabalhadores mais ligados à produção nas siderúrgicas, sendo que deve afetar tanto a vida de relações, o afe to, como também os parâmetros fisiológicos das pessoas. 


\section{TABELA 7}

Comparaçōes entre os niveis de pressão arterial na admissão com os do último exame, segundo os grupos de ocupaçôes - SNVR, 1979 - Somente para aqueles com 5 anos e mais de trabalho

\begin{tabular}{|c|c|c|c|c|c|c|}
\hline \multirow{2}{*}{ Grupos de Ocupações } & \multicolumn{2}{|c|}{$\begin{array}{c}\text { Sistólica } \\
\text { Admissão/Ult. Exame } \\
(\mathrm{mmHg} / \mathrm{mmHg})\end{array}$} & \multirow[b]{2}{*}{ Signific. } & \multicolumn{2}{|c|}{$\begin{array}{r}\text { Diastólica } \\
\text { dmissão/Ult. Exame } \\
(\mathrm{mmHg} / \mathrm{mmHg})\end{array}$} & \multirow[b]{2}{*}{ Signific. } \\
\hline & $\begin{array}{l}<140 /<140 \\
\geqslant 140 /<140\end{array}$ & $\begin{array}{l}<140 / \geqslant 140 \\
\geqslant 140 / \geqslant 140\end{array}$ & & $\begin{array}{l}<90 /<90 \\
\geqslant 90 /<90\end{array}$ & $\begin{array}{l}<90 / \geqslant 90 \\
\geqslant 90 / \geqslant 90\end{array}$ & \\
\hline 1 - Administrativas e técnico-científicas & $\begin{array}{l}8 \\
1\end{array}$ & $\begin{array}{l}1 \\
1\end{array}$ & NS & $\begin{array}{l}8 \\
1\end{array}$ & $\begin{array}{l}1 \\
1\end{array}$ & NS \\
\hline 2 - Escritório e almoxarifado & $\begin{array}{r}31 \\
3\end{array}$ & 9 & NS & $\begin{array}{r}32 \\
1\end{array}$ & 10 & $\mathrm{p}<0,01$ \\
\hline 3 - Laboratório e controle de qualidade & $\begin{array}{r}14 \\
-\end{array}$ & $\begin{array}{l}2 \\
1\end{array}$ & NS & $\begin{array}{r}12 \\
1\end{array}$ & $\begin{array}{l}3 \\
1\end{array}$ & NS \\
\hline 4 - Maquinaria e específicas da produção & $\begin{array}{r}34 \\
5\end{array}$ & $\begin{array}{l}3 \\
5\end{array}$ & NS & $\begin{array}{r}29 \\
1\end{array}$ & $\begin{array}{r}16 \\
1\end{array}$ & $\mathrm{p}<0,001$ \\
\hline 5 - Eletro-mecânicas de manutenção & $\begin{array}{r}41 \\
3\end{array}$ & $\begin{array}{l}7 \\
1\end{array}$ & NS & $\begin{array}{r}42 \\
1\end{array}$ & $\begin{array}{l}8 \\
1\end{array}$ & $\mathrm{p}<0,05$ \\
\hline 6 - Construção civil & $\begin{array}{l}7 \\
3\end{array}$ & $\begin{array}{l}3 \\
6\end{array}$ & NS & 9 & $\begin{array}{l}8 \\
2\end{array}$ & $\mathrm{p}<0,01$ \\
\hline 7 - Transportes e comunicações & $\begin{array}{r}18 \\
1\end{array}$ & $\begin{array}{l}3 \\
5\end{array}$ & NS & $\begin{array}{r}18 \\
1\end{array}$ & $\begin{array}{l}6 \\
2\end{array}$ & NS \\
\hline 8 - Prestação de serviços e segurança & $\begin{array}{l}7 \\
1\end{array}$ & $\begin{array}{l}2 \\
1\end{array}$ & NS & $\begin{array}{l}8 \\
1\end{array}$ & 2 & NS \\
\hline $\begin{array}{l}9 \text { - Não-qualificadas (ajudantes, } \\
\text { serventes e trabalhadores) }\end{array}$ & $\begin{array}{r}43 \\
6\end{array}$ & $\begin{array}{r}18 \\
8\end{array}$ & $\mathrm{p}<0,05$ & 48 & $\begin{array}{r}21 \\
6\end{array}$ & $\mathrm{p}<0,001$ \\
\hline
\end{tabular}

Em nossa amostra não dispomos de nenhuma medida direta do stress emocional provocado pelo trabalho. Entretanto, seria difícil argumentar que o stress pudesse ser a causa comum de variações positivas significativas, especialmente da pressão diastólica, em trabalhadores da construção civil, das ocupações específicas da produção siderúrgica e dos não-qualificados, assim como nos funcionários de escritório e almoxarifado (tabelas 4 e 5 ).

É preciso ressaltar que as maiores variações de status de pressão diastólica, do grupo normotenso para o de pressão mais elevada, ocorrem justamente nos grupos da construção civil, de maquinaria e especificas da produção, e, dos nãoqualificados (tabelas 6 e 7). Praticamente a metade dos trabalhadores da construção civil apresentou status de pressão mais elevada, tanto sistólica como diastólica, no último exame de rotina. Estas foram as proporções mais altas de hipertensos encontradas em todos os grupos.

Outros trabalhos procuraram demonstrar a relação de fatores ambientais com a pressão arterial. O rurdo parece estar ligado, de forma positiva, principalmente com a diastólica ${ }^{5,10,27}$. Foram encontradas prevalências mais altas de hipertensão associadas com o calor no ambiente de trabalho ${ }^{16,17}$. Também o trabalho em linhas de montagem e o que produz vibrações já foram relacionados com o aumento da presão sistólica ${ }^{10}$. 
Todos esses três fatores estão presentes no ambiente de trabalho siderúrgico, afetando mais diretamente os funcionários de maquinaria e espećficicas da produção, os nãoqualificados (ajudantes e serventes), e os das ocupações eletro-mecanicas de manutenção. Pelo menos o primeiro fator, o ruído, é integrante do ambiente de trabalho da construção civil.

Um outro fator que poderia ter alguma relevância para explicar as variações de pressão é a ingestão freqüente de bebida alcoઠlica, cuja conexão com níveis mais elevados de pressão já foi apontada ${ }^{2,23}$, inclusive em Volta Redonda ${ }^{15}$. Já que a maioria das variaçôes significativas ocorreu na pressão diastólica, poderia ser lembrado ainda o papel desempenhado pela obesidade ${ }^{3,12,23}$. Porém, mais uma vez não pudemos analisar estas relações, que exigiriam medidas feitas em cada um dos indivíduos.

Um raro estudo que compara duas medidas de pressão tomadas de cada pessoa, com um intervalo de tempo prolongado entre elas, cerca de três anos, foi feito para estudar a influência da aposentadoria sobre a evolução dos níveis tensionais ${ }^{9}$. Este estudo comparou o grupo de pessoas que se aposentaram durante $o$ intervalo entre as medidas com $o$ daquelas que continuaram trabalhando. Os resultados apontaram um aumento discreto da sistolica dos aposentados e um decréscimo da diastólica dos que continuaram trabalhando. Estes achados parecem discordar dos nossos em relação ao comportamento da pressão diastólica, porém é preciso chamar a atenção para o fato de que a faixa etária deste estudo era de 55 a 74 anos, portanto, a amostra examinada era muito mais velha do que a nossa, em que o indivíduo mais velho tinha 60 anos de idade. $\mathrm{E}$, como é comum acontecer em qualquer tipo de população, as médias de diastólica são crescentes até os 55 anos, de forma discreta se comparadas com o crescimento da sistólica, e, a partir desta idade, passam a ser declinantes ${ }^{7,13}$.

Vimos, ainda, nos resultados da análise de nossos dados, que as variações de pressão foram examinadas, segundo dois critérios: um variável, que dependia da pressão inicial de cada pessoa (tabelas 4 e 5), e outro, fixo, com pontos de corte definidos para sistólica e diastólica (tabelas 6 e 7). A semelhança entre os resultados correspondentes, como já foi mencionado, foi quase perfeita, com exceção do que ocorreu com os funcionários de escritório e almoxarifado e de laboratório e controle de qualidade, com pelo menos cinco anos de atividade, cujas variações de sistólica para mais foram significativas segundo o critério variável, mas não de acordo com o critério fixo. Isto, sem dúvida, se deu porque eram justamente estes os dois grupos onde se concentraram os menores valores de pressão sistólica no 
exame inicial. Assim, apesar de que a maioria significativa das pessoas destes grupos sofreram elevação das suas sistolicas entre os exames inicial e final, isto não foi suficiente para que mudassem significativamente de status de sistólica, ou seja, ultrapassassem o ponto de corte de $140 \mathrm{mmHg}$ no último exame. Por outro lado, estes tambem eram os grupos mais jovens, em média, na época do exame de admissão (tabela 2).

É preciso comentar o que aconteceu com o grupo ocupacional socialmente mais privilegiado, o de administradores e profissionais técnico-científicos. Este é, certamente, o grupo menos exposto a qualquer um dos fatores de risco apontados na literatura a respeito, sejam eles características individuais, fatores ambientais ou até mesmo stress emocional. Este grupo é aquele em que ocorrem variações em menor grau, entre todos, e sem nenhuma preferência de sentido, para qualquer uma das pressões. Deste modo, apesar deste grupo apresentar taxas de pressão alta, diastólica, das mais elevadas no exame de admissão, em relação aos demais conjuntos, por ser formado por pessoas mais velhas nesta época, isto não se repete na comparação com os demais grupos no último exame de rotina.

Finalmente, nâo levamos em consideração os efeitos de distorção provocados por tratamento anti-hipertensivo eventualmente administrado aos funcionários. Entretanto, supomos que estes possíveis efeitos não devem alterar expressivamente os resultados, uma vez que a empresa não executava um programa regular de prevenção e tratamento de hipertensão arterial.

\section{CONCLUSÃO}

Em que pese a limitação dos dados analisados, em rela. ção ao rigor da tomada das medidas, este estudo permite apontar alguns grupos de ocupações em que se observam alteraçð̃es significativas de pressão arterial. Os dados nos permitem isolar a ocupação como variável determinante da elevação da pressão arterial. Sabe-se que a um grupo ocupacłonal estão associados diversos fatores ambientais e hábitos de vida relacionados com alteraçōes dos níveis tensionais. Entretanto, os achados justificam uma abordagem diferenciada por parte dos serviços de saúde para esses grupos, compatível com os riscos especificos a que cada um está submetido.

\section{AGRADECIMENTOS}

Agradecemos ao Dr. Pedro Carlos Teixeira da Silva que por sua colaboração inestimável tornou possível 0 acesso 
ao arquivo de dados e ao Prof. Luis David Castiel, pelas suas doutas observações a respeito do bom uso da língua.

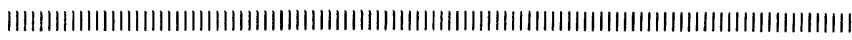

Data from the Medical Registry of the Companhia Sideruirgica Nacional de Volta Redonda (Rio de Janeiro, Brazil) drawn from a sample of 426 working nem from nine occupational categories are analysed. The aim of this study was to asses the evolution of BP from admittance to last periodical examination.

About $90 \%$ of the workers had 20 to 50 years at any of the measures. The mean interval between the measures was 13,5 years for those with at least 5 years in activity.

It was not possible to reject homogeneity of means of systolic or diastolic annual increments among occupational groups. In relation to the observed variations, significant systolic increases occurs only in the category of clerks and storekeepers as well as in that of laboratory and quality control workers, with at least 5 years in activity. Regarding diastolic pressure, occupational groups wich undergo significant increases were those of clerks and storekeepers, machinery and specific occupations of production, eletro-mechanics of maintenance, building construction, transportation/communications and non-skilled workers.

The shift from a status of normotension to the one of high BP (systolic 140 or diastolic $90 \mathrm{mmHg}$ ) was also analysed. Only non-skilled workers had significant changes in status of systolic. The results for the diastolic matched the ones observed for the increases.

It was noticeable the greatest increase of diastolic between the measures among building construction workers. Whereas managers and technical/scientifical personnel showed the smallest BP variations.

Environmental factors linked with BP elevation like noise, heat, assembly line and others are present in this steel industry.

The data points out a higher risk of PB elevation in particular occupational groups. These findings stress the recommendations of different oproaches by the mecical services in relation to this issue.

\section{REFERÊNCIAS BIBLIOGRÁFICAS}

1. ARAOJO, J.W.G. Hipertensão arterial em grupos sócio-ocupacionais de Volta Redonda - RJ. Rio de Janeiro, 1984. [Tese de Mestrado - ENSP/FIOCRUZ]. 
2. ARKWRIGHT, P.D.; BEILIN, L.J. \& ROUSE, I. Alcohol and blood pressure in a working population. Clin. Exp. Pharma. col. Physiol., 8 (5): 451-4, 1981.

3. ARO, S. Occupational stress, health-related behaviour, and bood pressure: a 5-year follow-up. Prev. Med. 13 (4):333-48, 1984.

4. ARO, S. Stress, morbidity, and health-related behaviour: a five-year follow-up among metal industry employees. Scand. J. Soc. Med., 9 (Suppl. 25).

5. BELLI, S.; SANI, L.; SCARFICCIA, G. \& SORRENTINO, R. Arterial hypertension and noise: a cross-sectional study. Am. J. Int. Med. 6 (1): 59-65, 1984.

6. CARVALHO, J.J.M.; SILVA, N.A.S.; OLIVEIRA, J.M.; ARGUELLES, E. \& SILVA, J.A.F. Pressão arterial e grupos sociais. Estudo pidemiológico. Arq. bras. Cariol., 40 (2): $115-20,1983$

7. COSTA, E.A. A Cross-sectional survey of blood pressure in Rio Grande do Sul, Brazil. London, 1981. [Tese de Doutorado - London School of Hygiene and Tropical Medicine].

8. COTTINGTON, E.M.; BROCK, B.M.; HOUSE, J.S. \& HAWTHORNE, V.M. Psychosocial factors and blood pressure in the Michigan Statewide Blood Pressure Survey. Am. J. Epidemiol. 121 (4): 515-29, 1985.

9. EKERDT, D.J.; SPARROW, D.; GLYNN, R.J. \& BOSSÉ, R. Change in blood pressure and total cholesterol with etirement. Am. J. Epidemiol., 120 (1):64-71, 1984.

10. FOURIAD, C.; JACQUINET-SALORD, M.C.; DEGOULET,P.; AIME, F.; LANG, T.; LAPRUGNE, J.; MAIN, J.; DECONOMOS, J. PHALENTE, J. \& PRADES, A. Influence of socioprofessional conditions on blood pressure levels and hypertension control. Am. J. Epidemiol., 120 (1): 72-86, 1984.

11. HADDAD, N. Inquérito epidemiológico sobre cardiopatias crônicas em um bairro de Ribeirão Preto - São Paulo - Brasil Arq. Hig. Saude publ., 32/33 (111/118): 27-77, 1967/68.

12. JACKSON, A.S.; SQUIRES, W.G. \& BUXTON, V.P. Evaluating of the Nasa/JSC Blood Pressure Screening Clinic. J. Occup. Med. 23 (6): 409-12, 1981.

13. KANNEL, W.B.; DAWBER, T.R.; KAGAN, A.; REVOTSKIE, N. \& STOKES, J. Factors of risk in the development of coronary heart disease, six-year follow-up experience. Ann. Int. Med. 55 (1): 33.50, 1961 .

14. KLEIN, C.H. Hipertensão arterial em estratos geo-econômicos do Rio Grande do Sul. Rio de Janeiro, 1981. [Tese de Mestrado - ENSP/FIOCRUZ].

Cadernos de Saúde Pública, R.J., 2(2):212-226, abr/jan, 1986 
15. KLEIN, C.H. \& ARAOJO, J.W.G. Fumo, bebida alcoólica, migração, instrução, ocupação, agregação familiar e pressão arterial em Volta Redonda, Rio de Janeiro, Cad. Saúde públ., 1 (2): 160-76, 1985.

16. KLOETZEL, K. ANDRADE, A.E. de \& FALLEIROS, J. Relationship between hypertension and prolongued exposure to heart. J. Occup. Med., 15 (6): 878-85, 1973.

17. LUND-LARSEN, P.G. \& DAHLBERG, B.E. Blood pressure in aluminium plant workers employed in high and in normal temperature divisions of Ardal and Sunndal Verk A/S. Tidsskr. Nor. Laegeforen, 101 (33): 1982-5, 1981.

18. MARMOT, M.G.; ROSE, G.; SHIPLEY, M. \& HAMILTON, P.J.S. Employment grade and coronary heart disease in british civil servants. J. Epidemiol. Community Health, 32 (4): 244-9, 1978.

19. MELhADO, J.C.; MOSA, A.A.P. \& DINE, J.M. Avaliação de níveis de pressão arterial em operários da construção civil. Rev. bras. Saúde. Ocup., 12 (45): 67-73, 1984.

20. MUSTACHI, P. The interface of the work environment and hypertension. Med. Clin. North Am., 61 (3): 531-45, 1977.

21. MYERS, J.E.; WHITE, N. \& CORNELL, J.E. Prevalence of hypertension in semiskilled manual workers. South $A f r$. Med. 62 (24): 894-8, 1982.

22. OAKES, T.W.; SYME, S.L.; FELDMAN, R.; FRIEDMAN, G.D.; SIEGELAUB, A.B. \& COLLEN, M.F. Social factors in newly discovered elevated blood pressure. J. Health \& Soc. Behav., 14: 198-204, 1973.

23. OPIT, L.J.; OLIVER, R.G. \& SALZBERG, M. Occupation and blood pressure. Med. J. Aust., 140 (13): 760-4, 1984.

24. RIBEIRO, D. Hypertension among female workers in São Paulo, Brazil. Predictors and joint effects. Hypertension, 5 (6III): V-144-V-148, 1983.

25. ROCA-CUSACHS, A.; CAMINAL, J.; MARINÉ, L.; SANS, S.; SCHMIDT, C.; TOMÁS, L.; VARELA, S. \& VINTRO, I.B. Epidemiologia de la hipertension arterial en una población laboral (I). Prevalencia, variación y variabilidad y estudio de factores presuntamente relacionados. Rev. Esp. Cardiol. 37 (6): 389-93, 1984.

26. SIEGEL, S. Nonparametric statistics for the behavioral sciences. New York, Mc Graw Hill Co., 1956.

27. TALBOTT, E.; HELMKAMP, J. MATTHEWS, K.; KULLER, L.; COTTINGTON, E. \& REDMOND, G. Occupational noise exposure noise-induced hearing loss, and the epidemiology of high blood pressure. Am. J. Epidemiol, 121 (4): $501-14,1985$.

28. WORLD HEALTH ORGANIZATION. Arterial hypertension. Washington, 1978. [Technical reports, 628]. 